\title{
Robust wavefront dislocations of Friedel oscillations in gapped graphene
}

\author{
Shu-Hui Zhang ${ }^{1 \S}$ Jin Yang ${ }^{2 \S}$, Ding-Fu Shao ${ }^{3}$, Zhenhua $\mathrm{Wu}^{4}$, and Wen Yang ${ }^{2}$ \\ ${ }^{1}$ College of Mathematics and Physics, Beijing University of Chemical Technology, Beijing, 100029, China \\ ${ }^{2}$ Beijing Computational Science Research Center, Beijing 100193, Chind \\ ${ }^{3}$ Department of Physics and Astronomy and Nebraska Center for Materials and Nanoscience, \\ University of Nebraska, Lincoln, Nebraska 68588-0299, USA and \\ ${ }^{4}$ Key Laboratory of Microelectronics Devices and Integrated Technology, \\ Institute of Microelectronics of Chinese Academy of Sciences, 100029, People's Republic of China
}

\begin{abstract}
Friedel oscillation is a well-known wave phenomenon, which represents the oscillatory response of electron waves to imperfection. By utilizing the pseudospin-momentum locking in gapless graphene, two recent experiments demonstrate the measurement of the topological Berry phase by corresponding to the unique number of wavefront dislocations in Friedel oscillations. Here, we study the Friedel oscillations in gapped graphene, in which the pseudospin-momentum locking is broken. Unusually, the wavefront dislocations do occur as that in gapless graphene, which expects the immediate verification in the current experimental condition. The number of wavefront dislocations is ascribed to the invariant pseudospin winding number in gaped and gapless graphene. This study deepens the understanding of correspondence between topological quantity and wavefront dislocations in Friedel oscillations, and implies the possibility to observe the wavefront dislocations of Friedel oscillations in intrinsic gapped two-dimensional materials, e.g., transition metal dichalcogenides.
\end{abstract}

Since the seminal discovery of graphene ${ }^{1}$, two-dimensional materials have attracted wide interest because of their novel physics and great potential applications ${ }^{2}$. Usually, twodimensional materials have high mobility, in which the antiparticles move ballistically and exhibit unconventional quantum tunneling and interference ${ }^{3-\underline{-8}}$. One can intentionally add one or two impurities to form the impurity-design system, this kind of system is charming because it is easily handled theoretically and experimentally, and then it can be regarded as the model system for the exploration of ballistic physics $\stackrel{9}{ }$. Experimentally, scanning tunneling spectroscopy (STM) is a proper tool for impurity-design system ${ }^{10}$. More interesting, the dimension of two-dimensional materials are very unique. On one hand, the bare surface properties of twodimensional materials are also the bulk properties in contrast to three-dimensional materials. On the other hand, different from the one-dimensional materials, two-dimension materials with two-dimensional parameter space is enough to evolve the global topological quantity ${ }^{11}$. Therefore, surface-sensitive STM measurement is promising to explore the topological physics of impurity-design two-dimensional materials.

Friedel oscillations (FO) is the quantum interference of electronic waves scattering by the imperfection in crystalline host materials 12 . Recently, STM has been demonstrated experimentally to measure the topological Berry phase $\pi$ of monolayer graphene by counting 2 wavefront dislocations in Friedel oscillations, which is induced by the intentional hydrogen adatom $\frac{13}{3}$. One subsequent experiment shows that for bilayer graphene FOs can exhibit 4, 2, or 0 wavefront dislocations explained by the $2 \pi$ Berry phase, the specific sublattice positions of the single impurity and the position of STM tip ${ }^{14}$. Electronic Berry phase as the intrinsic nature of the wave functions is defined in momentum space, which is responsible for many exotic electronic dynamics such as the index shift of quantum Hall effect in monolayer graphene ${ }^{15,16}$ and bilayer graphene $e^{17}$, Klein tunneling ${ }^{3.4}$ and the weak antilocalization 18 . The probe of Berry phase usually requires the magnetic field ${ }^{4,15,16,19}$. These two experiments not only do not need external magnetic field, but also realize the measurement of Berry phase in real space. However, two experiments both focus on the gapless cases for graphene and emphasize the relation between Berry phase and wavefront dislocation number. This attracts us to explore the effect of gap opening on the interference pattern of FOs.

Gap opening in graphene occurs in various different ways with the substrate coupling as a typical example ${ }^{20}$. Most experiments and devices are performed on the substratesupported graphene, in which lattice mismatch induced inversion symmetry breaking makes Berry phase unquantized multiple of $\pi^{21}$. As a result, gap opening should challenge the established correspondence relation between Berry phase and wavefront dislocation number in previous experiments ${ }^{13,14}$. In this study, we study the FOs in gapped graphene. Comparing to the gapless graphene, gapped graphene does not have pseudospin-momentum locking, this may prohibit the occurrence of characteristic interference structure (namely, wavefront dislocations) in FOs following the intuitive picture as suggested by the seminal work ${ }^{13}$. But the wavefront dislocations in FOs do emerge. Here, we explain the origin of wavefront dislocations by the invariant pseudospin winding number in gaped and gapless graphene, which can be regarded as an updated correspondence relation compatible with the previous experiments ${ }^{13,14}$. The wavefront dislocations in FOs of gapped graphene can be verified in the present experimental conditions, and this study helps deepen the understanding of topological physics reflected in the FOs of the impurity-design system.

Fig. 1 gives the schematic measurement of impurityinduced electronic density oscillation of monolayer graphene by STM. The present experimental technology allows one to intentionally introduce a single-atom vacancy on arbitrary sublattice site of graphene ${ }^{22}$, e.g., on the sublattice $A$ as shown by the red dot in Fig. 11a). Corresponding to the introduction of the single vacancy, FOs occur, and lead to the change of the space-resolved and energy-resolved local density of states (LDOS) $\delta \rho(\mathbf{r}, \varepsilon)^{23,24}$ : 


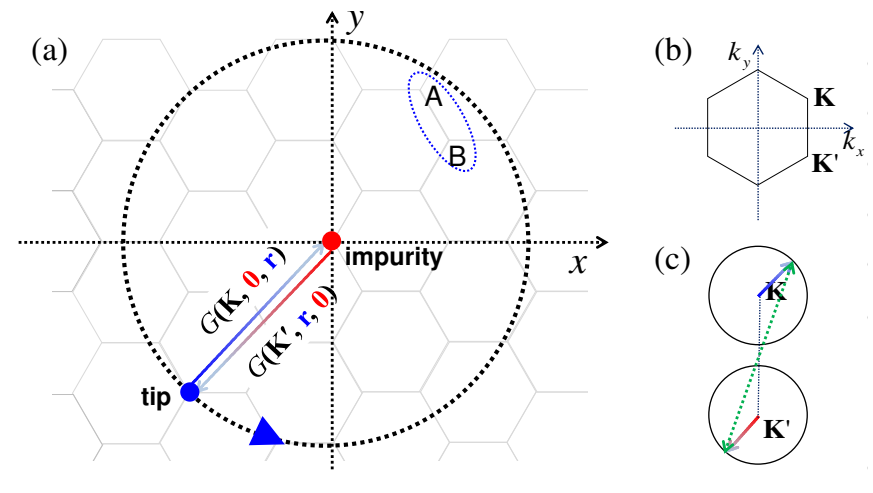

FIG. 1. Schematic measurement of electronic density oscillation by STM. (a) In real space atomic structure of graphene, there is a singleatom vacancy (red dot) on one sublattice site, by scanning the STM tip (blue dot) one can probe the vacancy-induced density oscillations which are contributed by the intravalley and intervalley scattering. Focusing on the intervalley scattering, we plot corresponding physical process, i.e., STM tip emits the electron waves in one valley $\mathbf{K}$, as shown by the propagator $G(\mathbf{K}, \mathbf{0}, \mathbf{r})$, scattering by the vacancy back to STM tip through the other valley $\mathbf{K}^{\prime}$, as shown by the propagator $G\left(\mathbf{K}^{\prime}, \mathbf{0}, \mathbf{r}\right)$. When the STM tip is shifted around the vacancy in real space as shown by the blue arrow along the circle, the contributing momentum states to the propagator change their momentum around $\mathbf{K}$ and $\mathbf{K}^{\prime}$ as shown in (c). (b) The Brillouin zone used to define $\mathbf{K}$ and $\mathbf{K}^{\prime}$. (c) Intervalley scattering (green double-arrowed line) in momentum space. The contributing momentum states in each valley are parallel to $\mathbf{r}$ and are denoted by using the same arrowed lines in (a).

$$
\delta \rho(\mathbf{r}, \varepsilon)=-\frac{1}{\pi} \operatorname{Im}[\operatorname{Tr} \delta \mathbf{G}(\mathbf{r}, \mathbf{r}, \varepsilon)],
$$

where $\delta \mathbf{G}=\mathbf{G}-\mathbf{G}^{0}$ represents the change of the total Green's function (GF) or propagator $\mathbf{G}$ incorporating the effect of the vacancy relevant to the bare propagator $\mathbf{G}^{0}$ of host system (i.e., graphene in Fig. 11a)), and has the form

$$
\delta \mathbf{G}\left(\mathbf{r}_{2}, \mathbf{r}_{1}, \varepsilon\right)=\mathbf{G}^{0}\left(\mathbf{r}_{2}, \mathbf{0}, \varepsilon\right) \mathbf{T G}^{0}\left(\mathbf{0}, \mathbf{r}_{1}, \varepsilon\right) .
$$

Here, the $T$-matrix approach is used to describe the effect of vacancy whose potential is simulated by $V_{0} \delta(\mathbf{r})$, and $T$-matrix is 25

$$
\mathbf{T}(\varepsilon)=\mathbf{V}\left[1-\int d^{2} \mathbf{k} \mathbf{G}^{0}(\mathbf{k}, \varepsilon) \mathbf{V}\right]^{-1} .
$$

In the $T$-matrix, $\mathbf{V}$ is usually a matrix and its form depends on the specific position of the vacancy, e.g., in Fig. 11a) (for the other vacancy configuration in supplementary materials), it is

$$
\mathbf{V}=\left[\begin{array}{cc}
V_{0} & 0 \\
0 & 0
\end{array}\right]
$$

For graphene, there are two Dirac valleys in the Brillouin zone $e^{\frac{8}{2}}$ (cf. Fig. 1(b)), $\mathbf{K}=\left(\frac{2 \pi}{3}, \frac{2 \pi}{3 \sqrt{3}}\right)$ and $\mathbf{K}^{\prime}=\left(\frac{2 \pi}{3},-\frac{2 \pi}{3 \sqrt{3}}\right)$, then $\delta \rho(\mathbf{r}, \varepsilon)$ are contributed by the intravalley and intervalley scattering. In graphene, the intravalley scattering contribution to $\delta \rho(\mathbf{r}, \varepsilon)$ has been well understood through many theoretical ${ }^{23,25-36}$ and experimental ${ }^{22,37-40}$ efforts, while intervalley scattering contribution attracts people's attention very recently due to its underlying topological nature $\mathrm{e}^{13,14,41}$. $\delta \rho(\mathbf{r}, \varepsilon)$ is measured easily by STM. FOs of $\delta \rho(\mathbf{r}, \varepsilon)$ are dominated by the backscattering events along the constant energy contour ${ }^{42.43}$. Focusing on the intervalley scattering, the corresponding physical process of Eq. 2 is shown in Fig. 11a), i.e., STM tip emits the electron waves from one valley $\mathbf{K}$, as shown by the propagator $G^{0}(\mathbf{K}, \mathbf{0}, \mathbf{r}, \varepsilon)$, scattering by the vacancy back to STM tip through the other valley $\mathbf{K}^{\prime}$, as shown by the propagator $G^{0}\left(\mathbf{K}^{\prime}, \mathbf{0}, \mathbf{r}, \varepsilon\right)$. Of course, the conjugate process also exists, i.e., the emission/scattering waves are from $\mathbf{K}^{\prime} / \mathbf{K}$ valley. Here, $G^{0}\left(\mathbf{K} / \mathbf{K}^{\prime}, \mathbf{0}, \mathbf{r}, \varepsilon\right)$ is the matrix element of $2 \times 2$ matrix $G^{0}$ and is associated with the valley momentum index. When the STM tip is shifted around the vacancy in real space as shown in Fig. 1 a), the contributing momentum states to the propagator change their momentum around $\mathbf{K}$ and $\mathbf{K}^{\prime}$ as shown in Fig. 1(c). As a result, the Berry phase defined in momentum space is measured by STM in real space, and the key is the pseudospin-momentum locking ${ }^{13}$. In contrast, we will show that the pseudospin winding number instead of Berry phase is measured by STM in gaped graphene without pseudospin-momentum locking.

The physics is essentially the same for gapped monolayer and bilayer graphene, described below using gapped monolayer as an example and the relevant results for gapped bilayer graphene in supplementary materials. The Hamiltonian of gaped monolayer graphene is $H_{0}=v_{F}\left(\eta \sigma_{x} k_{x}-\sigma_{y} k_{y}\right)+\Delta \sigma_{z}$. $H_{0}$ is expressed in the sublattice basis of $\{A, B\}$, then $\sigma_{x, y, z}$ is the Pauli matrix acting on the pseudospin space. $\pm \Delta$ is the staggered potential on the sublattice $A$ and $B$, which originates from the inversion symmetry breaking, e.g., by the proximity substrate $\mathrm{e}^{20,44}$. And $\eta= \pm 1$ is valley index for two inequivalent valleys in graphene, $v_{F}=3 / 2 a_{0} t_{0}$ with $a_{0}$ being the carbon-carbon bond length and $t_{0}$ being the nearest-neighbor hopping energy, and $a_{0} / t_{0}$ is used as the length/enegy unit in our convention $\stackrel{8}{*}$. The energy spectrum and the spinor wavefunction are, respectively,

$$
E_{\xi}(\mathbf{k})=\xi v_{F} \sqrt{\delta^{2}+k^{2}}
$$

and

$$
\psi_{\xi, \mathbf{k}}=\frac{1}{\sqrt{1+k^{2} /\left(\epsilon_{\xi}+\delta\right)^{2}}}\left[\begin{array}{c}
1 \\
\frac{\eta k_{x}-i k_{y}}{\epsilon_{\xi}+\delta}
\end{array}\right],
$$

where the reduced quantities are defined as $\delta=\Delta / v_{F}, \epsilon_{\xi}=$ $E_{\xi} / v_{F}$ with $\xi= \pm 1$ for the conduction and the valence band. The GF in momentum space is defined as $G^{0}(\mathbf{k}, \varepsilon) \equiv(z-$ $\left.H_{0}\right)^{-1}$, and it is

$$
\mathbf{G}^{0}(\mathbf{k}, \varepsilon)=\frac{1}{z^{2}-\Delta^{2}-k^{2} v_{F}^{2}}\left[\begin{array}{cc}
z+\Delta & \eta v_{F} k e^{i \eta \theta_{k}} \\
\eta v_{F} k e^{-i \eta \theta_{k}} & z-\Delta
\end{array}\right] .
$$

Here, $z=\varepsilon+i 0^{+}$with $0^{+}$for the retarded properties of GF and the Fermi level $\varepsilon$ is assumed in the conduction band for brevity. Performing the Fourier transformation to the momentum space GF, we express the real space $\mathrm{GF}^{45}$ as $\mathbf{G}^{(0)}(\mathbf{K}, \mathbf{r}, \varepsilon)=-e^{i \mathbf{K} \cdot \mathbf{r}} /\left(2 v_{F}\right)^{2} \mathbf{G}^{(0)}(\mathbf{r}, \varepsilon)$ with 


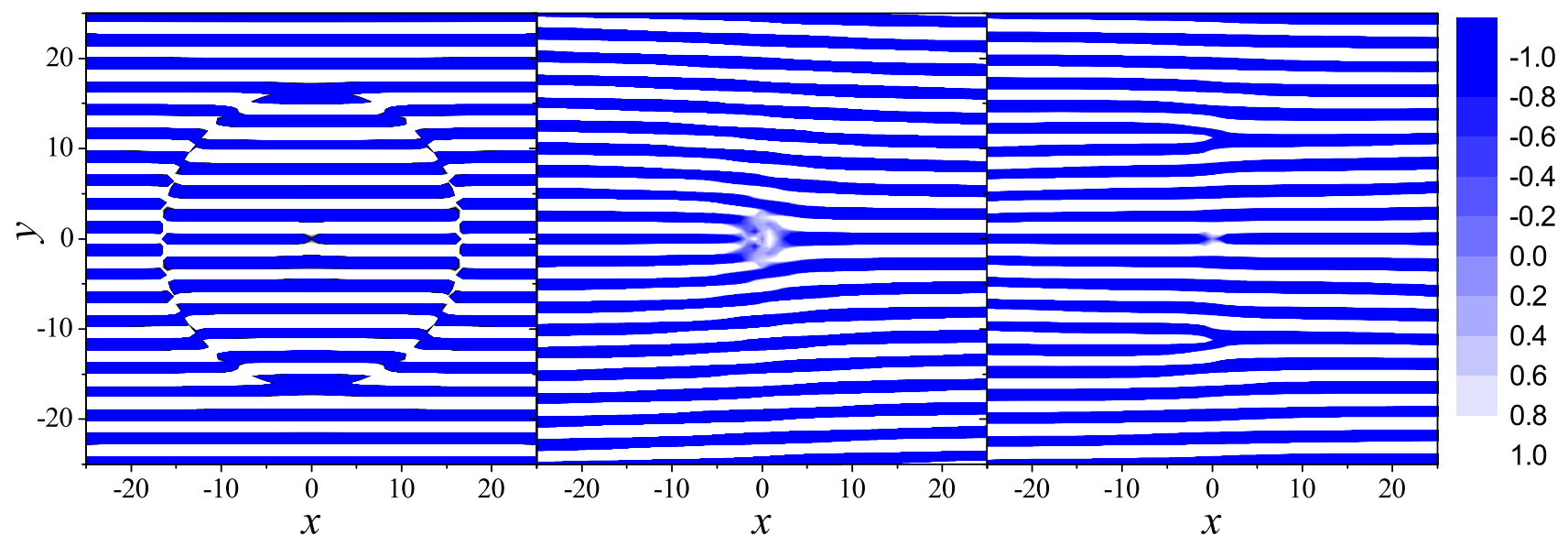

FIG. 2. Friedel oscillations pattern around a single-atom vacancy at the origin. Intervalley scattering contribution to density oscillations $\delta \rho_{A}(\mathbf{r}, \varepsilon)$ and $\delta \rho_{B}(\mathbf{r}, \varepsilon)$ on sublattice $A$ (left panel) and on sublattice $B$ (middle panel), respectively. And the sum $\delta \rho_{A}(\mathbf{r}, \varepsilon)+\delta \rho_{B}(\mathbf{r}, \varepsilon)$ as total electronic density modulation (right panel). Referring to 13 , the energy is integrated over energy up to Fermi energy. Here, Fermi level $\varepsilon=0.11 t_{0} \simeq 0.4 \mathrm{eV}, \Delta=0.017 t_{0} \simeq 50 \mathrm{mev}$, the color scale is normalized by a numerical factor $C \times 10^{-3}$.

$$
\mathbf{G}^{(0)}(\mathbf{r}, \varepsilon)=\left[\begin{array}{cc}
i \varepsilon_{+} H_{0}(u) & \eta \sqrt{\varepsilon_{+} \varepsilon_{-}} H_{1}(u) e^{i \eta \theta_{r}} \\
\eta \sqrt{\varepsilon_{+} \varepsilon_{-}} H_{1}(u) e^{-i \eta \theta_{r}} & i \varepsilon_{-} H_{0}(u)
\end{array}\right]
$$

where $H_{j}$ is $j$-th order Handel function of the first kind, $\varepsilon_{ \pm}=$ $\varepsilon \pm \Delta, u=r \sqrt{\varepsilon_{+} \varepsilon_{-}} / v_{F}$, and $r\left(\theta_{r}\right)$ is the module (azimuthal angle) of $\mathbf{r}$. Concentrating on the intervalley contribution, the change of LDOS is

$$
\delta \rho(\Delta \mathbf{K}, \mathbf{r}, \varepsilon)=\delta \rho_{A}(\mathbf{r}, \varepsilon)-\delta \rho_{B}(\mathbf{r}, \varepsilon),
$$

where the sublattice-resolved LDOS are

$$
\begin{aligned}
& \delta \rho_{A}(\mathbf{r}, \varepsilon)=C \operatorname{Im}\left[t(\varepsilon) H_{0}^{2}(u) \varepsilon_{+}^{2}\right] \cos (\Delta \mathbf{K} \cdot \mathbf{r}), \\
& \delta \rho_{B}(\mathbf{r}, \varepsilon)=C \operatorname{Im}\left[t(\varepsilon) H_{1}^{2}(u) \varepsilon_{+} \varepsilon_{-}\right] \cos \left(\Delta \mathbf{K} \cdot \mathbf{r}-\Delta \eta \theta_{r}\right) .
\end{aligned}
$$

Here, $\Delta \mathbf{K}=\mathbf{K}-\mathbf{K}^{\prime}, \Delta \eta=\eta-\eta^{\prime}, C=1 /\left(8 v_{F}^{4}\right)$ and $t(\varepsilon)=$ $V_{0} /\left[1-V_{0} G_{A A}^{(0)}(0, \varepsilon)\right]$ is the matrix element of $T$-matrix induced by the vacancy on the sublattice $A$.

Nontrivially, Eq. 2 for the LDOS contributed by the intervalley scattering has the identical form as that in gapless graphene ${ }^{13}$ and it reproduces the result of gapless case when $\Delta \rightarrow 0$. For the vacancy on the sublattice $A, \delta \rho_{A}(\mathbf{r}, \varepsilon)$ is trivial. $|\Delta \eta|=2$ for the intervalley scattering, then the phase of $\delta \rho_{B}(\mathbf{r}, \varepsilon)$ is singular at $\mathbf{r}=0$. By shifting STM tip around the vacancy, i.e., $\theta_{r}$ is rotated by $2 \pi$, there should be 2 additional wavefronts in the Friedel oscillation pattern of $\delta \rho_{B}(\mathbf{r}, \varepsilon)^{13}$. In Fig. 2 focusing on the intervalley scattering contribution, we show electronic density oscillations around a single-atom vacancy $\delta \rho_{A}(\mathbf{r}, \varepsilon)$ and $\delta \rho_{B}(\mathbf{r}, \varepsilon)$ on sublattice $A$ (left panel) and on sublattice $B$ (middle panel), respectively. And the sum $\delta \rho_{A}(\mathbf{r}, \varepsilon)+\delta \rho_{B}(\mathbf{r}, \varepsilon)$ as total electronic density modulation (right panel). As expected, $\delta \rho_{A}(\mathbf{r}, \varepsilon)$ exhibit the normal oscillating wavefronts perpendicular to $\Delta \mathbf{K}$ with a wavelength (a)

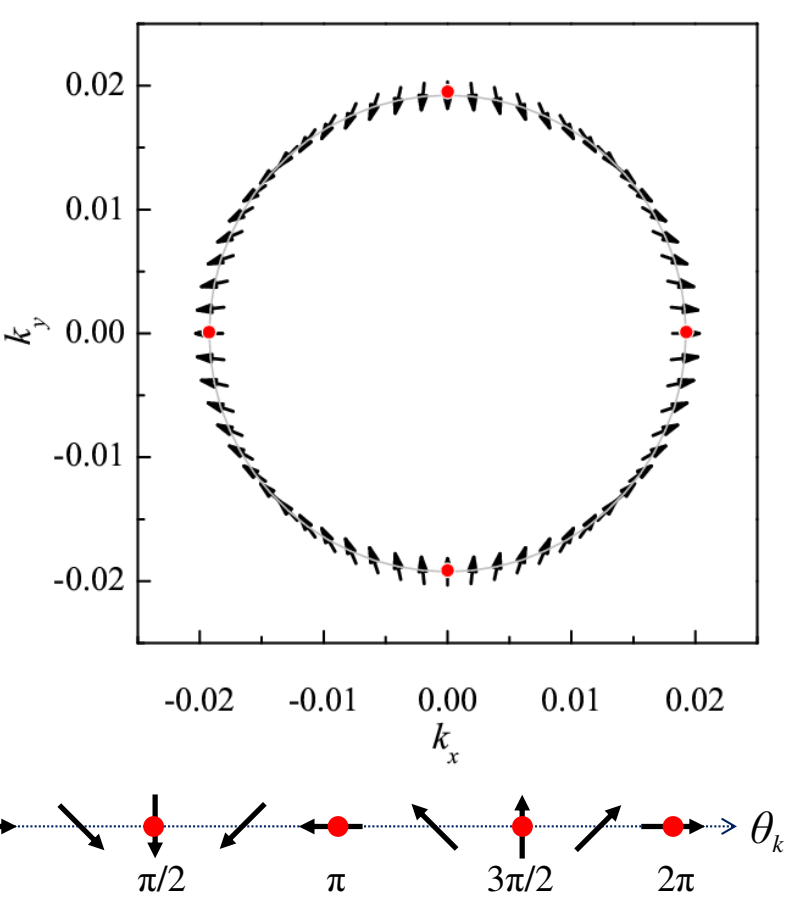

FIG. 3. Pseudospin texture pattern. (a) Pseudospins on the constant energy contour. (b) Pseudospin as the function of $\theta_{k}$. Here, $\Delta=50$ $\mathrm{meV}, \varepsilon=100 \mathrm{meV}$ and $\eta=1$.

$\lambda_{\Delta \mathbf{K}}=2 \pi /|\Delta \mathbf{K}|=2.60 a_{0} \approx 3.69 \stackrel{\circ}{a}$, and does not display any topological feature. $\delta \rho_{B}(\mathbf{r}, \varepsilon)$ gives two wavefront dislocations at $\mathbf{r}=0$, which accommodates for the phase accumulated along the contour enclosing the singular point of the phase $\theta_{r} \stackrel{46}{=}$. In the total electronic density modulation (cf. right panel of Fig. 2), $\delta \rho_{A}(\mathbf{r}, \varepsilon)$ only shifts the position of dislocations from $\mathbf{r}=0$ along the direction parallel to $\Delta \mathbf{K}$, but does not change the shape and the number of dislocations ${ }^{13}$.

The number 2 of additional wavefronts is regarded as the 
signature of the Berry phase $\pi$ of graphene. However, the correspondence between Berry phase and the wavefront dislocation number fails since the Berry phase is unquantized multiple of $\pi$ in gapped graphene. Back to Fig. 1(c), in which we do not follow the references ${ }^{13,14}$ to plot the momentum-resolved pseudospin direction, but it still shows synchronous motion of the dominant scattering state contributing to FOs in momentum space and STM tip in real space, e.g., the scattering state rotates clockwise/counterclockwise on the constant energy contour when STM tip is shifted clockwise/counterclockwise around the vacancy. The scattering state can be used to define the pseudospin vector $\mathbf{s}=\left(s_{x}, s_{y}\right)$ where

$$
\begin{aligned}
& s_{x}=\left\langle\psi_{\xi, \mathbf{k}}\left|\sigma_{x}\right| \psi_{\xi, \mathbf{k}}\right\rangle=\frac{2 \eta k_{x}\left(\epsilon_{\xi}+\delta\right)}{\left(\epsilon_{\xi}+\delta\right)^{2}+k^{2}}, \\
& s_{y}=\left\langle\psi_{\xi, \mathbf{k}}\left|\sigma_{y}\right| \psi_{\xi, \mathbf{k}}\right\rangle=-\frac{2 k_{y}\left(\epsilon_{\xi}+\delta\right)}{\left(\epsilon_{\xi}+\delta\right)^{2}+k^{2}} .
\end{aligned}
$$

Due to $s_{x} \sim k_{x}$ and $s_{y} \sim k_{y}$, Eq. 11 gives 4 fixing points with the momentum azimuthal angle $\theta_{k}=0, \pi / 2, \pi$, and $2 \pi$, at which the pseudospin directions are fixed. This feature has no dependence on $\delta$, then is robust to the gap opening. To show more visually, we arbitrarily choose a set of parameters to plot the pseudospin texture in Fig. 3. With the scattering state moving on the constant energy contour, the pseudospin direction twists continuously in Fig. 3) (a). Clearly, there are 4 fixing points shown by the red dots (cf. Fig. 3) (a)), which implies the invariant pseudospin winding number. Referring to Fig. 3 (b), when $\theta_{k}$ evolves from 0 to $2 \pi$, the pseudospin rotates by $2 \pi$ corresponding to the winding number 1 . Alternatively, the invariant pseudospin winding number can also be shown mathematically ${ }^{47}$. To rewrite the gapped Hamiltonian of monolayer graphene into the form:

$$
H_{0}(\mathbf{k})=\left|E_{\xi}(\mathbf{k})\right|\left[\begin{array}{cc}
\cos \alpha & \sin \alpha e^{-i \phi} \\
\sin \alpha e^{i \phi} & -\cos \alpha
\end{array}\right],
$$

where the azimuthal $\alpha(\mathbf{k})$ and polar $\phi(\mathbf{k})$ angles on the Bloch sphere are defined as $\cos \alpha=\Delta /\left|E_{\xi}(\mathbf{k})\right|, \sin \alpha=f(\mathbf{k}) /\left|E_{\xi}(\mathbf{k})\right|$ with $f(\mathbf{k})=v_{F}\left(\eta k_{x}+i k_{y}\right)$ and $\phi \equiv-\operatorname{Arg} f$. As a result, we obtain the Berry phase along a closed Fermi surface:

$$
\gamma(C)=\oint_{C} d \mathbf{k} \cdot \mathbf{A}_{\xi}=\pi W_{C}\left[1-\frac{\Delta}{\left|E_{\xi}(\mathbf{k})\right|}\right] .
$$

Here, we have used the Berry connection $\mathbf{A}_{\xi}=i\left\langle u_{\mathbf{k}, \xi} \mid \nabla_{\mathbf{k}} u_{\mathbf{k}, \xi}\right\rangle=$ $-\xi \sin ^{2} \frac{\alpha}{2} \nabla_{\mathbf{k}} \phi$. Most importantly, the winding number is introduced

$$
W_{C} \equiv-\xi \oint_{C} \frac{d \phi}{2 \pi}=\eta \xi
$$

While the Berry phase of Eq. 13 has the $\Delta$ dependence and becomes unquantized multiple of $\pi, W_{C}$ of Eq. 14 with the absolute value 1 is topologically invariant in gapless and gapped graphene. As a result, the 2 wavefront dislocations also exist in gapped graphene, which should be ascribed to the invariant winding number.

Here, we firstly discuss the experimental implication of our theoretical discovery. When $\Delta \rightarrow 0$ for gapless graphene, one can say that the Berry phase and winding number are equivalent, then Berry phase is measured by the wavefront dislocations 13,14 . But a supplementary experiment is necessary to confirm the gapless nature of graphene sample. In previous two experiments ${ }^{13,14}$, there is no such supplementary experiment and they do not perform the quantitative comparison between experiments and simulations for the FOs, which both prohibit the discovery of the possible gap opening. The quantitative calculations of FOs, especially properly incorporating the strength and range of impurity potential, is rather important and is worth of further simulations, e.g., by using density functional calculations ${ }^{48}$. Also, this experimental discussions are also applicable to one recent theoretical proposal, which uses the presence or absence of wavefront dislocations in FOs to distinguish the incompatible low-energy models for twisted bilayer graphene ${ }^{49}$. In light of present experimental technology, we expect the immediate verification of our theoretical prediction in gapped graphene. In addition, our results may stimulate the theoretical and experimental research interest on the FOs in intrinsic gapped two-dimensional materials, e.g., transition metal dichalcogenides 50,51 .

The robustness of the wavefront dislocations of FOs to the gap opening dictates the potential applications in graphenebased pseudospintronics ${ }^{52}$. Each imperfection can be regarded as one vortex, and the sublattice dependence of wavefront dislocation help define the vortex and antivortex since the orientations of the tripod shape of the imperfection on two sublattices are different ${ }^{13}$. Then, we can use the vortex/antivortex as $0 / 1$ to construct the memory device. And the the writing of vortex-based memory device can be performed by using STM to shift the imperfection from one sublattice to the other one ${ }^{22}$, while its reading is realized by scanning the imperfection-induced FOs to characterize the orientations of the tripod shape of the imperfection. One recent experiment ${ }^{41}$ studies the effect of spacing on the wavefront dislocation of two vortices, and shows the irrelevant vortices on the several nanometer scale which favors the high-density of vortex-based memory device. This implies the great potential of impurity-design graphene in pseudospintronics.

In summary, we have studied the Friedel oscillation in gapped graphene. Although the pseudospin-momentum locking is broken, the wavefront dislocations still emerge in intervalley scattering contributed Friedel oscillations. We establish the correspondence between the invariant winding number instead of the Berry phase and the number of wavefront dislocations in gapped graphene, which can be verified by the present experimental technology. This study is helpful to the understanding of correspondence between topological quantity and wavefront dislocations in Friedel oscillations, and broadens the range of materials to observe the wavefront dislocations of FOs, e,g, in transition metal dichalcogenides 50,51 . 


\section{ACKNOWLEDGEMENTS}

The authors thank Dr. Li-Kun Shi for helpful discussions. This work was supported by the National Key R\&D Program of China (Grant No. 2017YFA0303400), the National Natural Science Foundation of China (NSFC) (Grants No. 11774021 and No. 11504018), and the NSAF grant in NSFC (Grant No. U1930402). We acknowledge the computational support from the Beijing Computational Science Research Center (CSRC).
* shuhuizhang@mail.buct.edu.cn

† wenyang@csrc.ac.cn

$\$$ \$These two authors contributed equally to this work.

${ }^{1}$ K. S. Novoselov, A. K. Geim, S. V. Morozov, D. Jiang, Y. Zhang, S. V. Dubonos, I. V. Grigorieva, and A. A. Firsov, Science 306, 666 (2004).

2 C. Liu, H. Chen, S. Wang, Q. Liu, Y.-G. Jiang, D. W. Zhang, M. Liu, and P. Zhou, Nature Nanotechnology 15, 545 (2020).

${ }^{3}$ M. I. Katsnelson, K. S. Novoselov, and A. K. Geim, Nat. Phys. 2, 620 (2006).

4 A. F. Young and P. Kim, Nat. Phys. 5, 222 (2009).

5 V. V. Cheianov, V. Fal'ko, and B. L. Altshuler, Science 315, 1252 (2007).

${ }^{6}$ C. W. J. Beenakker, R. A. Sepkhanov, A. R. Akhmerov, and J. Tworzydło, Phys. Rev. Lett. 102, 146804 (2009).

7 Z. Wu, F. Zhai, F. M. Peeters, H. Q. Xu, and K. Chang, Phys. Rev. Lett. 106, 176802 (2011).

8 A. H. Castro Neto, F. Guinea, N. M. R. Peres, K. S. Novoselov, and A. K. Geim, Rev. Mod. Phys. 81, 109 (2009).

9 M. Settnes, S. R. Power, D. H. Petersen, and A.-P. Jauho, Phys. Rev. Lett. 112, 096801 (2014).

${ }^{10}$ D. N. Basov, M. M. Fogler, A. Lanzara, F. Wang, and Y. Zhang, Rev. Mod. Phys. 86, 959 (2014).

11 C. Dutreix, M. Bellec, P. Delplace, and F. Mortessagne, Wavefront dislocations reveal insulator topology (2020), 2006.08556.

12 J. Friedel, The London, Edinburgh, and Dublin Philosophical Magazine and Journal of Science 43, 153 (1952).

13 C. Dutreix, H. González-Herrero, I. Brihuega, M. I. Katsnelson, C. Chapelier, and V. T. Renard, Nature 574, 219 (2019).

14 Y. Zhang, Y. Su, and L. He, Phys. Rev. Lett. 125, 116804 (2020).

15 K. S. Novoselov, A. K. Geim, S. V. Morozov, D. Jiang, M. I. Katsnelson, I. V. Grigorieva, S. V. Dubonos, and A. A. Firsov, Nature 438, 197 (2005).

16 Y. Zhang, Y.-W. Tan, H. L. Stormer, and P. Kim, Nature 438, 201 (2005).

17 K. S. Novoselov, E. McCann, S. V. Morozov, V. I. Falko, M. I. Katsnelson, U. Zeitler, D. Jiang, F. Schedin, and A. K. Geim, Nature Physics 2, 177 (2006).

18 X. Wu, X. Li, Z. Song, C. Berger, and W. A. de Heer, Phys. Rev. Lett. 98, 136801 (2007).

19 D. Xiao, M.-C. Chang, and Q. Niu, Rev. Mod. Phys. 82, 1959 (2010).

20 A. Chaves, J. G. Azadani, H. Alsalman, D. R. daCosta, R. Frisenda, A. J. Chaves, S. H. Song, Y. D. Kim, D. He, J. Zhou, et al., npj 2D Materials and Applications 4, 29 (2020).

21 W. Yao, D. Xiao, and Q. Niu, Phys. Rev. B 77, 235406 (2008).

22 Gonzalez-Herrero, Hector, Brihuega, Ivan, Ugeda, Miguel, M., Gomez-Rodriguez, Jose, and M. and, Science 352, 437 (2016).

${ }^{23}$ C. Bena, Phys. Rev. Lett. 100, 076601 (2008).

24 Y.-L. Zou, J. Song, C. Bai, and K. Chang, Phys. Rev. B 94, 035431 (2016).

25 C. Dutreix and M. I. Katsnelson, Phys. Rev. B 93, 035413 (2016).
26 V. V. Cheianov and V. I. Falko, Phys. Rev. Lett. 97, 226801 (2006).

27 E. H. Hwang and S. Das Sarma, Phys. Rev. Lett. 101, 156802 (2008).

28 T. Pereg-Barnea and A. H. MacDonald, Phys. Rev. B 78, 014201 (2008).

29 C. Bena, Phys. Rev. B 79, 125427 (2009).

${ }^{30}$ F. M. D. Pellegrino, G. G. N. Angilella, and R. Pucci, Phys. Rev. B 80, 094203 (2009).

31 A. Bácsi and A. Virosztek, Phys. Rev. B 82, 193405 (2010).

32 G. Gomez-Santos and T. Stauber, Phys. Rev. Lett. 106, 045504 (2011).

33 J. A. Lawlor, S. R. Power, and M. S. Ferreira, Phys. Rev. B 88, 205416 (2013).

${ }^{34}$ M. Settnes, S. R. Power, D. H. Petersen, and A.-P. Jauho, Phys. Rev. B 90, 035440 (2014).

35 M. Settnes, S. R. Power, J. Lin, D. H. Petersen, and A.-P. Jauho, Phys. Rev. B 91, 125408 (2015).

36 T. M. Rusin and W. Zawadzki, Phys. Rev. B 97, 205410 (2018).

37 Rutter, M. G., J. N. Crain, Guisinger, P. N., T., N. First, P., Stroscio, and A. J., Science 317, 219 (2007).

${ }^{38}$ I. Brihuega, P. Mallet, C. Bena, S. Bose, C. Michaelis, L. Vitali, F. Varchon, L. Magaud, K. Kern, and J. Y. Veuillen, Phys. Rev. Lett. 101, 206802 (2008).

39 P. Mallet, I. Brihuega, S. Bose, M. M. Ugeda, J. M. GomezRodriguez, K. Kern, and J. Y. Veuillen, Phys. Rev. B 86, 045444 (2012).

${ }^{40}$ K. W. Clark, X.-G. Zhang, G. Gu, J. Park, G. He, R. M. Feenstra, and A.-P. Li, Phys. Rev. X 4, 011021 (2014).

41 Y. Zhang and L. He, arXiv (2020), 2008.03956.

42 P. T. Sprunger, L. Petersen, E. W. Plummer, E. Laegsgaard, and F. Besenbacher, Science 275, 1764 (1997).

43 S.-H. Zhang, D.-F. Shao, and W. Yang, Journal of Magnetism and Magnetic Materials 491, 165631 (2019).

44 S. Y. Zhou, G.-H. Gweon, A. V. Fedorov, P. N. First, W. A. deHeer, D.-H. Lee, F. Guinea, A. H. Castro Neto, and A. Lanzara, Nature Materials 6, 770 (2007).

45 J.-J. Zhu, D.-X. Yao, S.-C. Zhang, and K. Chang, Phys. Rev. Lett. 106, 097201 (2011).

46 J. F. Nye, M. V. Berry, and F. C. Frank, Proceedings of the Royal Society of London. A. Mathematical and Physical Sciences 336, 165 (1974).

47 J. N. Fuchs, F. Piechon, M. O. Goerbig, and G. Montambaux, The European Physical Journal B 77, 351 (2010).

${ }^{48}$ K. Noori, S. Y. Quek, and A. Rodin, Phys. Rev. B 102, 195416 (2020).

49 V. O. T. Phong and E. J. Mele, Phys. Rev. Lett. 125, 176404 (2020).

${ }^{50}$ Q. H. Wang, K. Kalantar-Zadeh, A. Kis, J. N. Coleman, and M. S. Strano, Nature Nanotechnology 7, 699 (2012).

51 J. R. Schaibley, H. Yu, G. Clark, P. Rivera, J. S. Ross, K. L. Seyler, W. Yao, and X. Xu, Nature Reviews Materials 1, 16055 (2016).

52 D. Pesin and A. H. MacDonald, Nat. Mater. 11, 409 (2012). 